\title{
Efecto de la cobertura vegetal de cuatro cultivos sobre la erosión del suelo
}

\author{
Effect of plant cover of four crops on soil erosion
}

\author{
José de Jesús Huerta-Olague ${ }^{1}$ † José Luis Oropeza Mota², *Rubén Dario Guevara Gutiérrez ${ }^{1}$ José \\ Donaldo Ríos Berber ${ }^{2}$, Mario Roberto Martínez Menes ${ }^{2}$, Oscar Arturo Barreto García ${ }^{1}$, José Luis \\ Olguín López ${ }^{1}$, Oscar Raúl Mancilla Villa ${ }^{3}$
}

\begin{abstract}
RESUMEN
En la presente investigación se evaluó el efecto del sistema aéreo de cuatro cultivos, tres de escarda (Zea mays L., Phaseolus vulgaris L. y Cucurbita pepo L.) y uno denso (Avena sativa L.) sobre la pérdida de suelo y escurrimiento superficial mediante lluvia simulada. La unidad de muestreo fueron 16 lotes de $1 \mathrm{~m}^{2}$ en el campo experimental de lomas de San Juan, de la Universidad Autónoma de Chapingo, Chapingo, Estado de México. El suelo Entisol migajón-arenoso, profundidad de 40cm y pendiente de $2 \%$. El crecimiento de la cobertura vegetal se cuantificó a través de fotografías digitales cada 15 días a partir de la siembra, captando la imagen multitemporal y suelo desnudo. El uso de coberturas vegetales manifiesta una relación exponencial negativa respecto a la pérdida de suelo; de los cultivos considerados en relación a la pérdida de suelo para reducir el proceso erosivo fue mayor en cebada > calabacita > frijol > maíz; la mejor correlación cobertura vegetal y reducción de la pérdida fue del cultivo de frijol seguido por calabacita, maíz y avena.

Palabras clave: Pérdida de suelo, escurrimiento superficial, cobertura vegetal.
\end{abstract}

\section{ABSTRACT}

In this research the effect of air system four crops, three of weeding (Zea mays $L$., Phaseolus vulgaris $L$. and Cucurbita pepo $L$.) and one dense (Avena sativa L.) on soil loss and runoff was assessed simulated surface by rain. The sampling unit were 16 lots of $1 \mathrm{~m} 2$ experimental field in the hills of San Juan, of the Autonomous University of Chapingo, Chapingo, State of Mexico. Entisol soil loam-sandy, depth of $40 \mathrm{~cm}$ and slope of $2 \%$. The growth of plant cover was quantified through digital photos every 15 days after planting, the multitemporal image capturing and bare soil. The use of mulches shows a negative exponential relationship to soil loss; of crops considered in relation to the loss of soil to reduce erosion process was higher in barley> squash> bean > corn; the best correlation vegetation cover and loss reduction bean crop was followed by squash, corn and oats.

Keywords: Loss of soil, surface runoff, vegetation cover.

\section{Introducción}

En el mundo existen más de 305 millones de hectáreas con alta degradación resultado principalmente de la alta deforestación de más de 12.3 millones de hectáreas anuales (Faminow, 1998). En México se reportan más de 50 millones de hectáreas con alta degradación, proceso acelerado por el cambio de uso del suelo, deforestación de más de 600,000 ha año ${ }^{-1}$ y agricultura de ladera (Lal,
2000). La degradación de los suelos es un proceso que puede ser inducido por el hombre que disminuye la capacidad actual y/o futura para sostener la vida humana y acelerado por efecto del sobrepastoreo, prácticas agrícolas superficiales y actividades industriales (Espinosa et al., 2011).

En áreas agrícolas el uso de las coberturas vegetales es sumamente importante ya que reducen la erosión del suelo, sobre todo en terrenos de fuertes pendientes (Loch, 2000). En áreas excluidas bajo

1 Universidad de Guadalajara, Centro Universitario de la Costa Sur, Departamento de Ecología y Recursos Naturales. Autlán de Navarro, Jalisco, México.

2 Colegio de Postgraduados, Texcoco, Edo. De México.

3 Universidad de Guadalajara, Centro Universitario de la Costa Sur, Departamento De Producción Agrícola.

* Autor por correspondencia: rguevara@cucsur.udg.mx

Fecha de Recepción: 7 octubre, 2016.

Fecha de Aceptación: 10 enero, 2018.

DOI: http://dx.doi.org/10.4067/S0718-34292018005000701. Publicado en línea: 06-septiembre-2018. 
condiciones de lluvia natural el efecto de la cobertura vegetal sobre el proceso erosivo, la combinación de un cultivo tupido o pasto en combinación con nopal resultó ser la práctica más efectivo en la reducción del proceso erosivo en comparación con monocultivos como pasto, nopal denso o éste último en combinación con coberturas bajas o medianas (Serna y Echavarría, 2002). Los cultivos tupidos de alta densidad (cebada, avena y trigo) presentan valores significativamente más eficientes en el control de la erosión y escurrimiento superficial (78\% y $65 \%$ respectivamente) en comparación a asociaciones de cultivos de escarda (maíz-frijol y maíz-haba) y éstos más eficientes cultivos únicos de escarda como maíz o frijol (Ríos y Martínez, 1990). Solano et al. (1990) para suelos de tepetate encontraron que las pérdidas anuales de suelo varían de acuerdo al uso del suelo y porcentaje de cobertura vegetal.

Flores et al. (2013) reconocen que el tipo de cobertura vegetal y manejo que se proporcione al cultivo, así como las características erosivas de las tormentas repercutirán en el incremento de la pérdida del suelo, principalmente en el inicio de la siembra o plantación del cultivo. La magnitud del proceso erosivo del suelo menciona Santacruz (2011) se incrementa por efecto de los cambios de uso de suelo, reconociendo el papel clave que juega la cobertura vegetal en el proceso erosivo de la lluvia; en bosques este cambio se estima hasta 9 veces sobre el $100 \%$ en incremento sobre la pérdida natural de suelo, la que se puede reducir hasta $60 \%$ mediante buenas prácticas agrícolas para zonas de temporal y riego. Francisco-Nicolás et al. (2006) identifican a las terrazas de muro vivo como prácticas eficientes en la reducción del escurrimiento superficial y pérdida de suelo hasta límites permisibles, situación que mejora la productividad a rendimientos aceptables.

En este trabajo se evaluó la capacidad de la cobertura vegetal en cuatro períodos de desarrollo de cultivos maíz (Zea mays L.), frijol (Phaseolus vulgaris L.), calabacita (Cucúrbita pepo L.) y avena (Avena sativa L.) para protección del suelo contra la fricción de escurrimientos superficiales y proceso erosivo, en lotes de escurrimiento, utilizando lluvia simulada a una intensidad constante de $80 \mathrm{~mm} \mathrm{~h}^{-1}$.

\section{Materiales y Métodos}

El estudio se realizó dentro del campo experimental "Lomas de San Juan" de laUniversidad Autónoma Chapingo, a $5 \mathrm{~km}$ sobre la carretera Chapingo-Tequexquinahuac, localizado en la región oriental de la Cuenca del Valle de México, a una altitud aproximada de 2,300 msnm, ubicado a 19²9'52.21" norte y $98^{\circ} 51^{\prime} 08.95$ " oeste del meridiano de Greenwich (Figura 1). El suelo es Lithic Ustorthent de acuerdo a la clasificación Soil Taxonomy (2006),

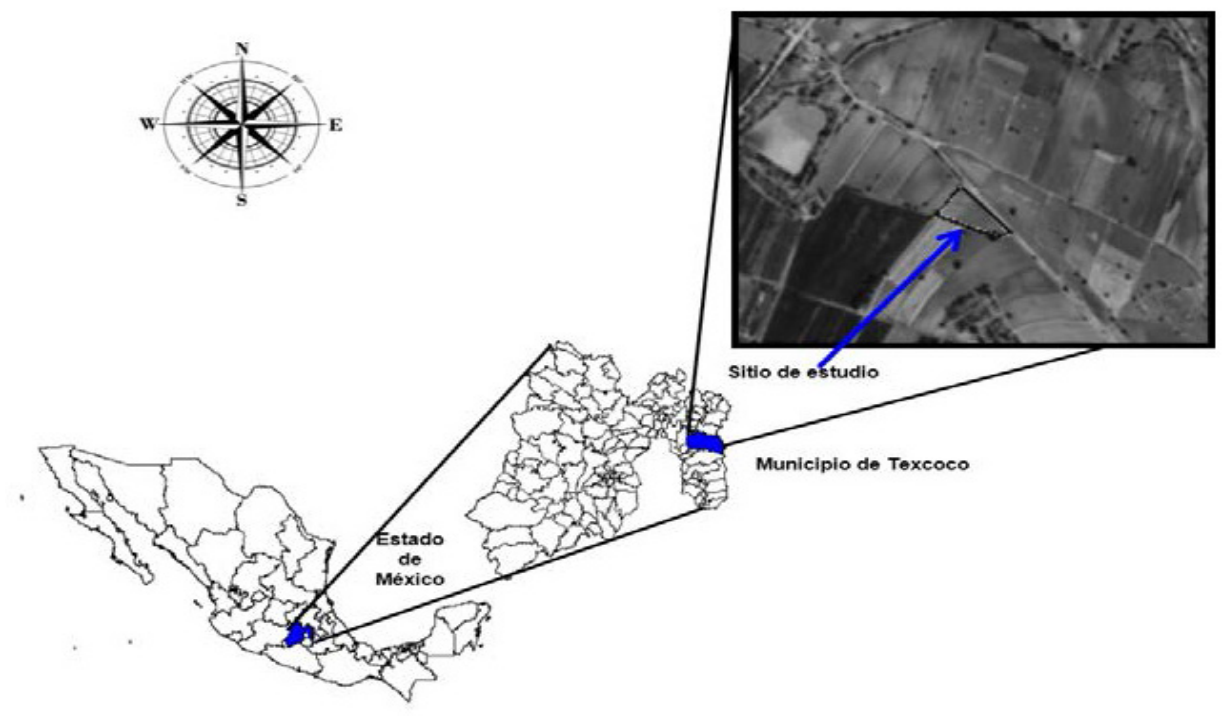

Figura 1. Ubicación de la zona de estudio, Lomas de San Juan, Valle Estado de México. 
profundidad de 25 a $40 \mathrm{~cm}$, textura migajón arenoso, pH de 7.3 y pendiente natural de $2 \%$. La precipitación media anual de $596.4 \mathrm{~mm}$ y temperatura media anual de $18^{\circ} \mathrm{C}$ con oscilación térmica de $\pm 3^{\circ} \mathrm{C}$.

\section{Métodos}

Se consideró la evaluación de la cobertura vegetal de cuatro cultivos (sin diseño experimental) sobre la ocurrencia de los escurrimientos superficiales y la pérdida de suelo, siendo maíz (Zea mays) y frijol (Phaseolus vulgaris L.) con separación entre hileras de $50 \mathrm{~cm}$ y $20 \mathrm{~cm}$ entre matas (sembrándose dos semillas por mata), calabacita (Cucurbita pepo L.) con separación entre hileras de 60 y entre matas de $40 \mathrm{~cm}$ (una semilla por mata), la avena (Avena sativa) fue sembrada al voleo. La unidad experimental empleada por cultivo fue una parcela de escurrimiento de $4 \mathrm{~m}^{2}$, subdividida en cuatro subparcelas de $1 \mathrm{~m}^{2}$, la densidad de siembra fue de manera tradicional a la establecida en la región (Tabla 1), la pendiente natural fue modificada a $10 \%$. La intensidad de lluvia simulada fue de $80 \mathrm{~mm} \mathrm{~h}^{-1}$ con un simulador tipo Purdue (Alcaláde-Jesús et al., 1998) con tiempo de simulación de una hora, a los 15, 30, 50 y 65 días de desarrollo del cultivo.

Tabla 1. Densidad de población, variedad y fertilización.

\begin{tabular}{lccc}
\hline \multicolumn{1}{c}{ Cultivo } & Variedad & Densidad & $\begin{array}{c}\text { Fertilización } \\
\text { Sulfato de amonio }\end{array}$ \\
\hline Maíz & San Josecito & 100,000 matas por ha & $116.83 \mathrm{~kg} \mathrm{ha}^{-1}$ \\
Frijol & Flor de junio & 100,000 matas por ha & Sin fertilización \\
Calabacita & Chey zucchini & 40,000 matas por ha & Sin fertilización \\
Avena & Juchitepec & $100 \mathrm{~kg} \mathrm{ha}^{-1}$ & $116.83 \mathrm{~kg} \mathrm{ha}^{-1}$ \\
\hline
\end{tabular}

Para obtener el volumen escurrido y la producción de sedimentos, las subparcelas se delimitaron con láminas de fierro enterradas $5 \mathrm{~cm}$ y sobresaliendo 5 $\mathrm{cm}$. En la parte baja de la subparcela se adaptó un colector metálico para recoger el escurrimiento superficial en cubetas de 8 litros de capacidad, a intervalos de tiempo de cinco minutos durante una hora, cada 15 días durante 65 días. La cuantificación del escurrimiento superficial en la subparcela se obtuvo mediante la siguiente relación.

$$
E s=\frac{10\left(\frac{P s h-P s S}{\rho_{a}}\right)}{A_{s u b}}
$$

Dónde: $E s=$ escurrimiento superficial $(\mathrm{mm})$, $P s h=$ volumen escurrido y el sedimento colectados (g), Pss = peso seco del sedimento (g), $\varrho a=$ densidad del agua $\left(1 \mathrm{~g} \mathrm{~cm}^{-3}\right), A s u b=$ área de la subparcela $\left(\mathrm{cm}^{2}\right), 10=$ factor de conversión a mm. La pérdida de suelo en cada subparcela Psh y Pss es obtenida en $\mathrm{Kg} \mathrm{m}^{-2}$.

La estimación del porcentaje de cobertura vegetal fue realizada cada 15 días mediante fotografías digitales de cada cultivo durante su desarrollo, obteniéndose el área de cobertura vegetal multitemporal y suelo descubierto (Figura 2). La fotografía $2 b$, fue recortada y sometida a un proceso de separación de tres bandas "RGB" (Red, Green, Blue) (Figura 3).

Para la edición de laimagen digital multitemporal se utilizó el software Corel Photo Paint X3 (Corel Corporation, 2005) generando imágenes en formato JPEG (Joint Photographic Experts Group) y transformadas a formato raster (RST) mediante el software IDRISI(Eastman, 2003). Una vezimportadas las imágenes RGB, se realizó la clasificación supervisada para generar puntos de control (campos de entrenamiento) generando polígonos de píxeles de características espectrales similares; definiendo las firmas espectrales para cada cultivo en diferentes periodos de desarrollo vegetativo, que indican la presencia o ausencia de vegetación (Figura 4).

Mediante el clasificador de firmas espectrales MAXLIKE y basado en el proceso estadístico de máxima verosimilitud, con probabilidad de asignación de 50\% para dos clases y $33 \%$ para tres (igual probabilidad) se compararon píxel por píxel en las tres bandas de cada clase, asignando a cada píxel la clase con mayor probabilidad de pertenencia (Figura 5). El porcentaje de cobertura vegetal fue calculado utilizando el modulo "área" de IDRISI, que contabiliza el número de píxeles asignados a cada clase. 


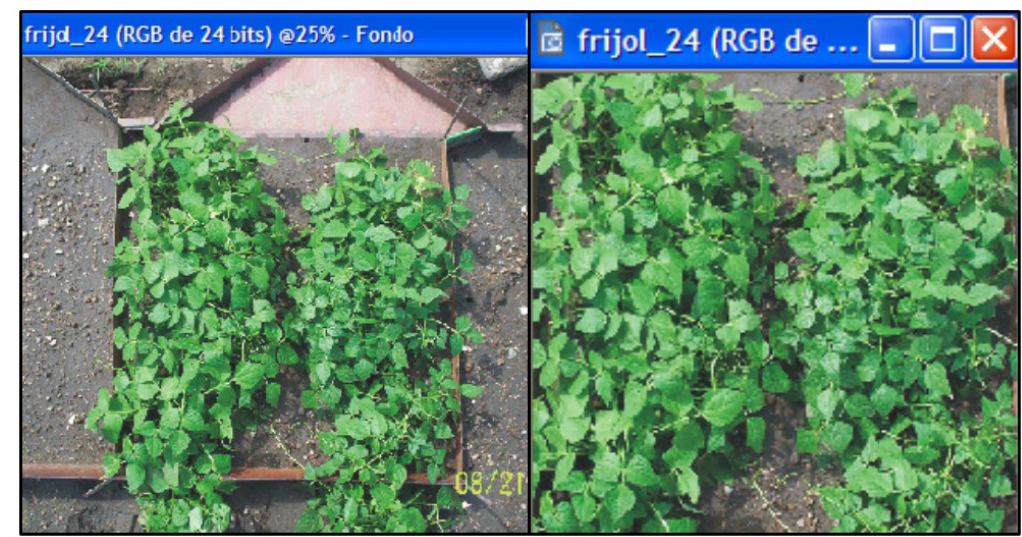

Figura 2. La imagen rotada y recortada. a) Fotografía original b) Recorte área de interés.

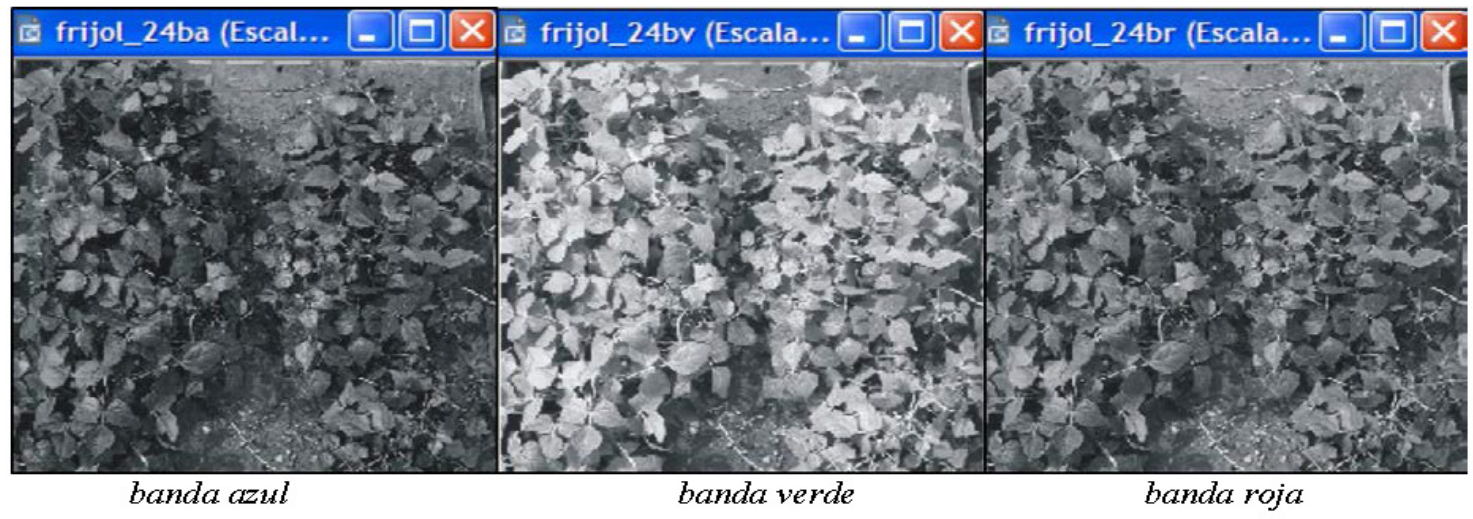

Figura 3. Fotografía separada en tres bandas.

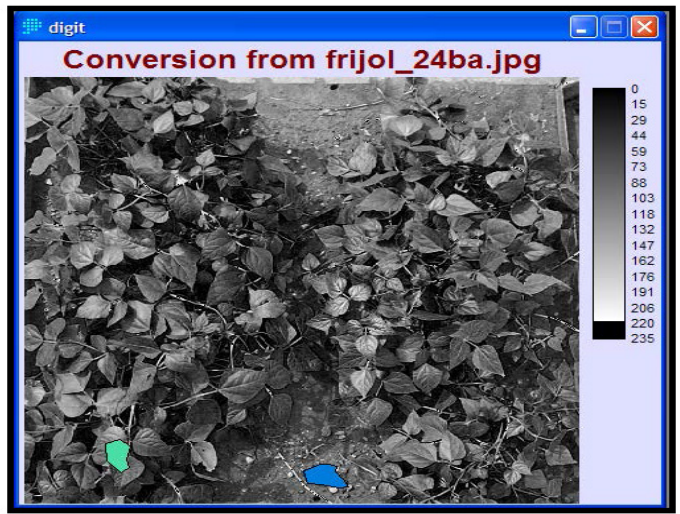

Figura 4. Polígonos de clases de firmas espectrales, suelo (azul) y vegetación (verde). 


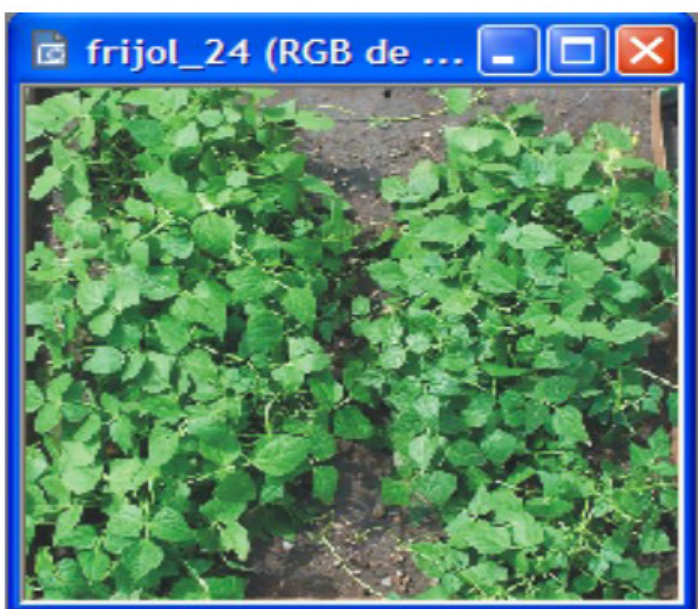

a) Fotografia original

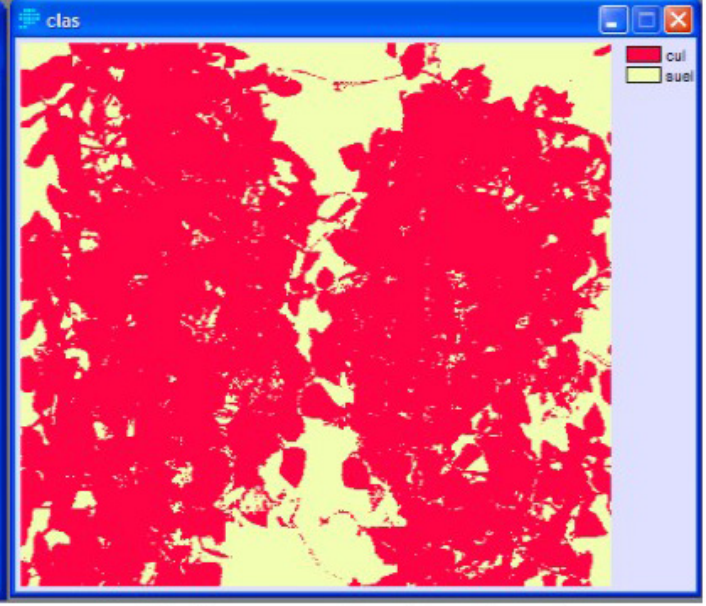

b) Fotografia clasificada

Figura 5. Resultado de la clasificación supervisada, vegetación (rojo) y suelo (amarillo).

\section{Resultados y discusión}

La comparación del desarrollo vegetal promedio de los cultivos de escarda y cultivo tupido fue para este último superior a $50 \%$ a los 15 días y cercano a $75 \%$ a los 30 días, para los cultivos de escarda fue hasta los 50 días cuando alcanzaron su máximo desarrollo de cobertura (65\%), respuesta reflejada mediante las estimación de las tasas de crecimiento (Figura 6). Estas diferencias se atribuyen a que los cultivos de escarda se siembran en hilera con una distancia entre matas y baja densidad de siembra, a diferencia del cultivo tupido sembrado al voleo con alta densidad de siembra (Figura 7). Comportamiento que hace suponer que los cultivos tupidos son más eficientes para reducir la energía cinética de las gotas de lluvia y del escurrimiento superficial, con ello la pérdida de suelo en comparación a los cultivos de escarda al menos durante los primeros 30 días de desarrollo.

\section{Escurrimiento superficial}

La respuesta del escurrimiento en cultivos de escarda (libres de maleza), muestra en maíz un comportamiento irregular registrando el máximo valor cuando se tiene el mayor porcentaje de cobertura $(\mathrm{R}=0.45)$ respuesta que se considera es efecto del manejo agronómico del cultivo y a su estructura física de la planta, ya que después de los 50 días de desarrollo, alcanzó una altura de $1.6 \mathrm{~m}$ interceptando la lluvia y descendiendo el agua entre el follaje del tallo hasta el suelo a poca velocidad, incrementando el escurrimiento superficial promedio a $49 \mathrm{~mm}$ con incremento promedio de $0.5 \%$, coeficiente de escurrimiento inicial de 0.48 incrementándose hasta 0.81 en su etapa final de desarrollo. Con respecto a los cultivos de frijol y calabacita $(\mathrm{R}=0.99$ y $\mathrm{R}=0.93$ respectivamente) así como avena $(\mathrm{R}=0.73)$, la respuesta fue inversamente proporcional en función del incremento de la cobertura vegetal a través del tiempo, reduciendo el escurrimiento en estos cultivos de escarda durante su desarrollo hasta $2.1 \%$ y $0.2 \%$ en avena. Resultados equiparables a los manifiestos por Osuna y Esquivel (1996), Quinton et al. (1997) y Loch (2000).

De los cultivos de escarda el frijol redujo en mayor proporción el escurrimiento superficial a partir de los 50 días de desarrollo y menor con respecto al cultivo tupido a partir de 65 días (Figura 8). Resultados que manifiestan diferencias significativas $(\alpha=0.05)$ entre cultivos; mediante la prueba de Tukey se observa que el maíz y la avena presentan diferencias significativas, enmarcando al frijol y calabacita con la mejor respuesta para reducir los escurrimientos superficiales. Rivera-Ruíz et al. (2012) identifican que los cultivos de escarda presentan una eficiencia de $40 \%$ en el control del escurrimiento a diferencia de los cultivos de tupidos como la avena que tiene una eficiencia de $60 \%$. 


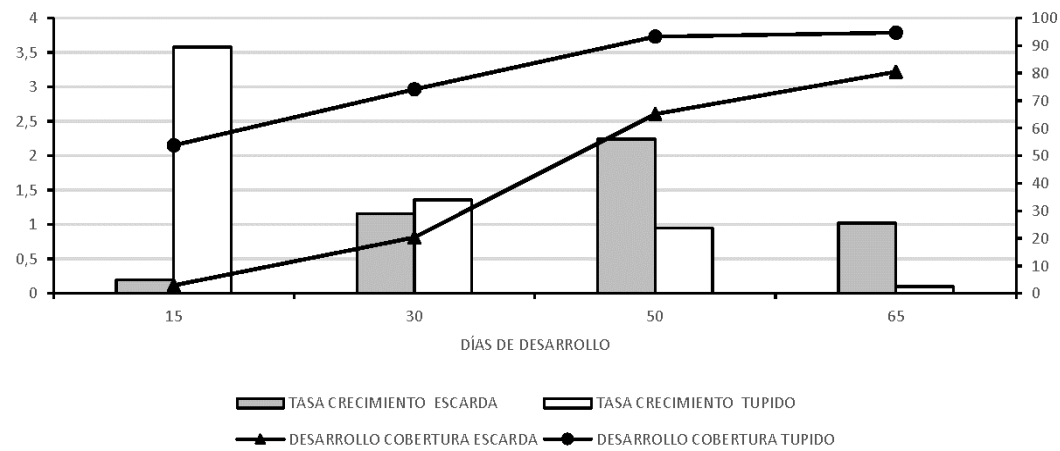

Figura 6. Desarrollo de cobertura vegetal y tasa de crecimiento de cultivos de escarda (valor promedio) y cultivo tupido.

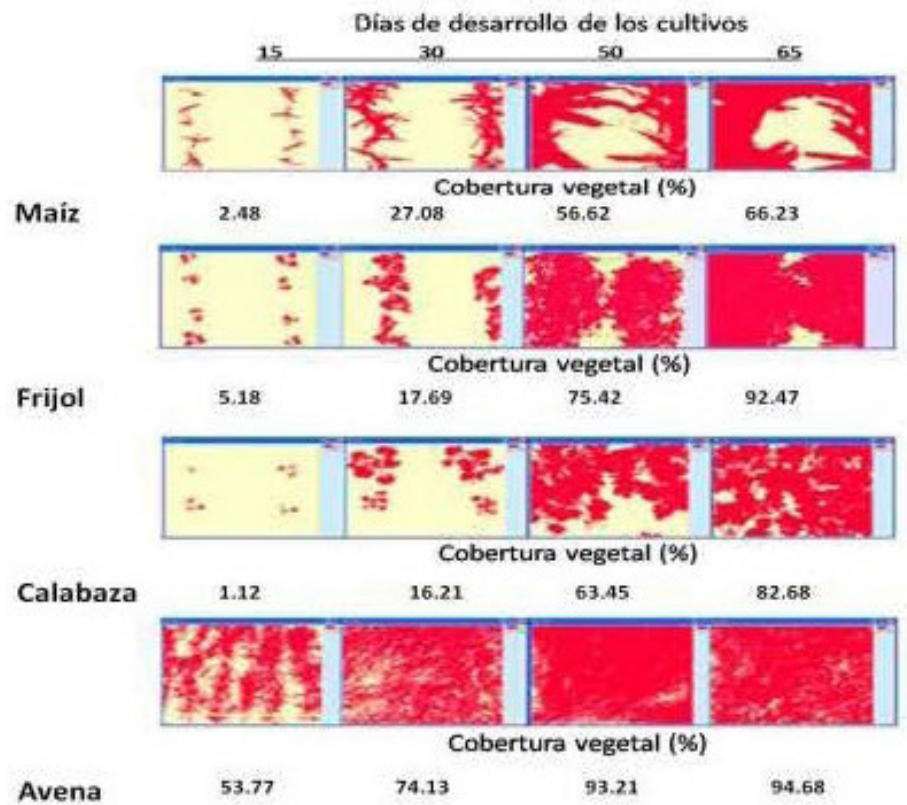

Figura 7. Evolución de la cobertura vegetal, durante el periodo experimental. Cobertura vegetal (rojo) y suelo (amarillo).

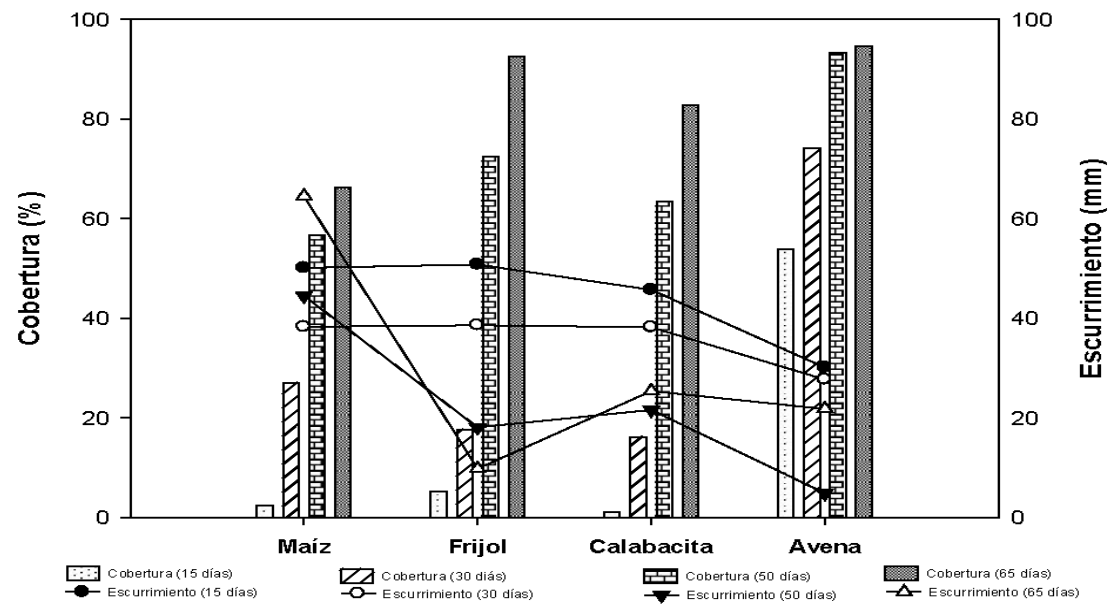

Figura 8. Eficiencia de la cobertura vegetal en el control del escurrimiento superficial. 


\section{Pérdida de suelo}

El efecto de las coberturas de los cultivos empleados registró una tendencia generalizada a reducir la pérdida de suelo hasta los primeros 50 días de desarrollo, en frijol y calabacita se mantuvo esta tendencia hasta los 65 días; en maíz y avena el proceso erosivo aumentó con respecto al incremento del escurrimiento superficial. Se destaca que todos los cultivos presentaron un modelo exponencial decreciente que permite a partir del porcentaje de cobertura vegetal predecir la pérdida de suelo. Gutiérrez y Hernández (1996) identifican que la vegetación es el principal factor que influye en la disminución del escurrimiento superficial y la pérdida de suelo.

El incremento de cobertura vegetal en el cultivo de maíz no implicó reducción del escurrimiento superficial pero sí la reducción en la pérdida de suelo $(\mathrm{R}=0.85)$. Hecho relacionado con la estructura de la planta y la presencia de gran cantidad de raíces fibrosas que amortiguan la energía cinética del agua (Figura 9a). La FAO (2000) reconoce que en cultivos de escarda en el período inicial (30 a 40 días de desarrollo) el maíz protege más al suelo de la erosión en comparación con la soya y algodón, posterior a este periodo ocurre una efectiva protección del suelo por estos cultivos.

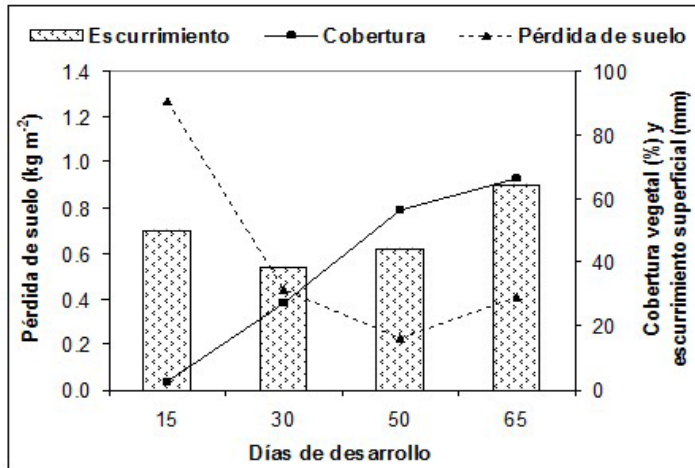

$a$

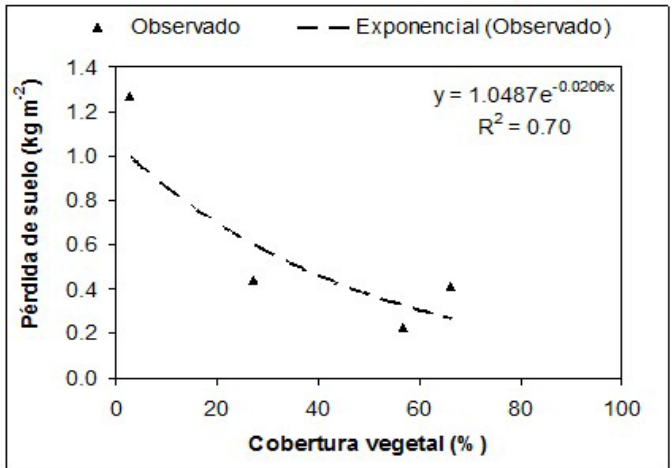

$b$

Figura 9. Pérdida de suelo y cobertura vegetal en maíz.

En los cultivos de frijol y calabacita al igual que en maíz se observó la reducción de la pérdida de suelo al incrementar la cobertura $(R=0.99$ y $\mathrm{R}=0.98$ ), respuesta que varía dependiendo de la densidad de siembra y su forma de crecimiento, lo que favorece la infiltración y reduce el escurrimiento superficial (Figura 10 y 11). Quinton et al. (1997) y Loch (2000) reportan resultados similares con $\mathrm{R}=0.78$ y $\mathrm{R}=0.99$. Carroll et al. (1997) y Carroll et al. (2000) determinaron que en cultivos agrícolas el incremento de la cobertura vegetal reduce progresivamente la erosión del suelo.

Finalmente en el cultivo de avena, se resalta su eficiencia para la protección del suelo debido al tipo de cobertura, densidad de siembra y sistema radical. En este cultivo a los 50 días del muestreo los resultados de pérdida de suelo fueron omitidos por considerar la existencia de un error de muestreo, no obstante su relación cobertura vegetal y pérdida de suelo presentó una relación de $\mathrm{R}=0.72$
(Figura 12). Estos resultados sustentan lo manifiesto por Wilcox y Wood (1989) en el sentido de que a mayor cobertura vegetal menor pérdida de suelo.

La repuesta generada por los cultivos empleados a la reducción de pérdida de suelo manifiesta una respuesta de mayor a menor en avena $>$ calabacita $>$ frijol > maíz, principalmente en los primeros 50 días de desarrollo de los cultivos. No obstante el cultivo de frijol presenta la mejor correlación entre el incremento de la cobertura vegetal y la reducción de la pérdida de suelo seguido por calabacita, maíz y avena.

Ramírez y Oropeza (2001), Loch (2000), Carroll et al. (2000), Betancourt et al. (2000), Belmonte et al. (1998) y Blackburn et al. (1992), señalaron que el uso de las coberturas vegetales en áreas agrícolas reducen la erosión de los suelos, ya que absorben la energía de la lluvia disminuyendo el impacto de las gotas de lluvia, aumentan la infiltración del agua y la rugosidad del terreno 


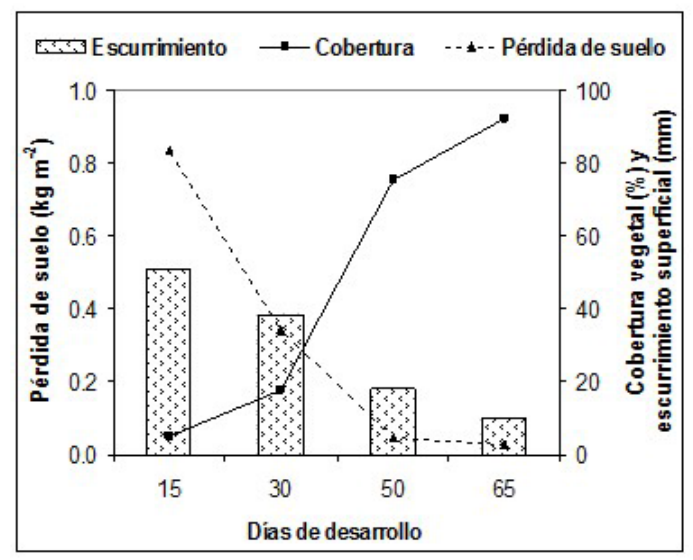

$a$

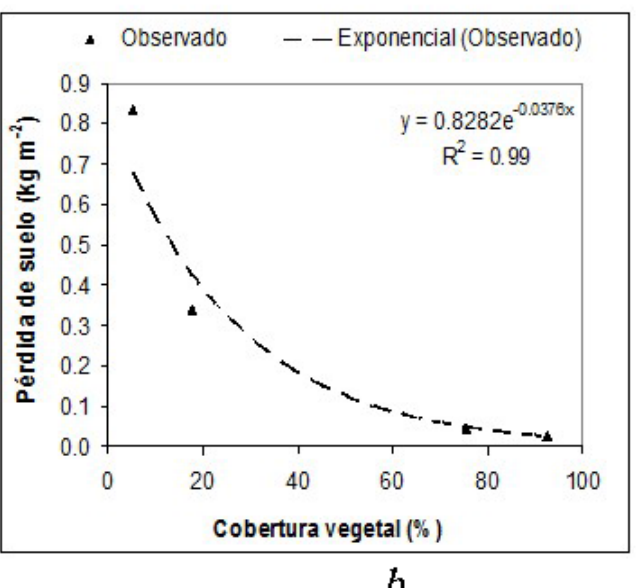

$b$

Figura 10. Pérdida de suelo y cobertura vegetal en frijol.

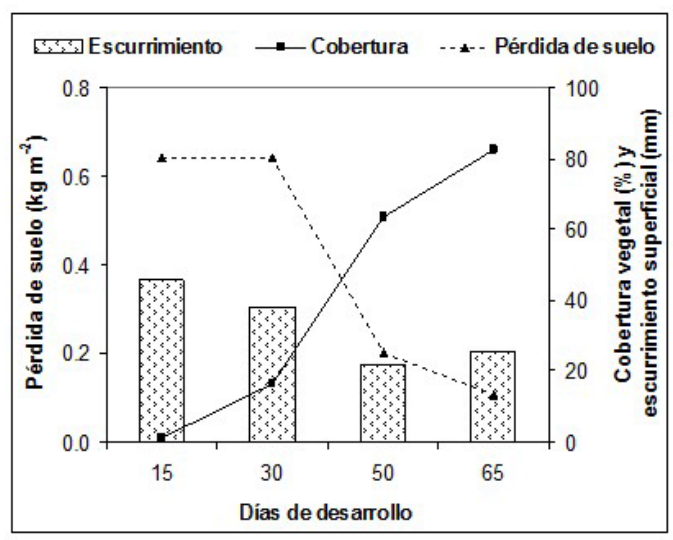

a

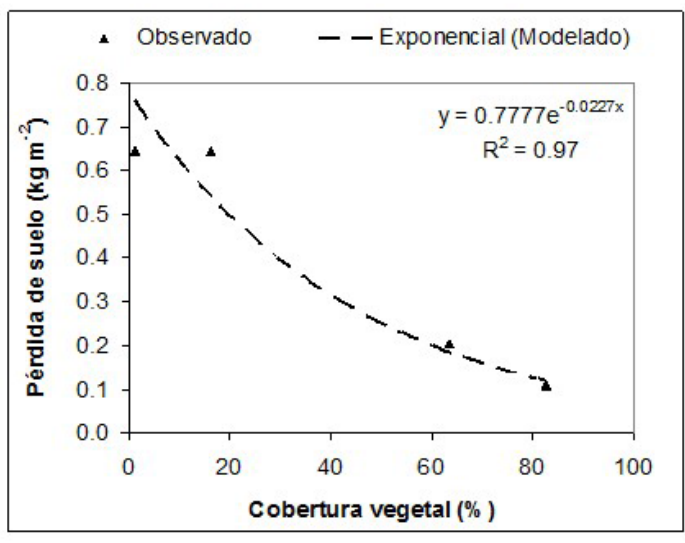

b

Figura 11. Pérdida de suelo y cobertura vegetal en calabacita.

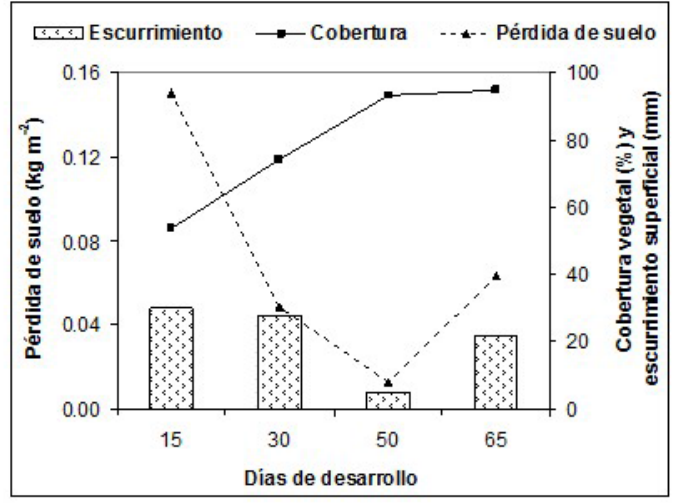

a

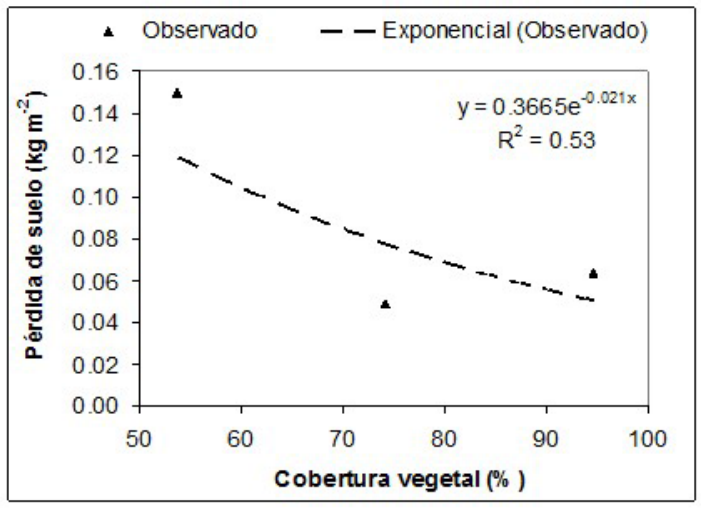

b

Figura 12. Pérdida de suelo y cobertura vegetal en avena. 
lo que reduce el escurrimiento y su capacidad de transporte.

\section{Conclusiones}

El uso de coberturas vegetales manifiesta una relación exponencial negativa respecto a la pérdida de suelo; el empleo de diferentes cultivos refleja diferencias significativas entre éstos para reducir esta pérdida. Los cultivos tupidos por el mayor porcentaje de cobertura vegetal en las diferentes etapas de desarrollo son reconocidos por su mayor capacidad para proteger al suelo en contra de los agentes erosivos en comparación con los cultivos de escarda. La estructura vegetal del cultivo del frijol es identificado con el mejor ajuste en la reducción del escurrimiento superficial, caracterizado por la variedad empleada, su hábito de crecimiento indeterminado semipostrado y follaje en contacto directo con el suelo, lo cual provoca mayor capacidad de intercepción de la lluvia y barrera eficaz al tránsito del escurrimiento superficial.

Es incuestionable el papel de la cobertura vegetal y las raíces de los cultivos para proveer protección a los terrenos agrícolas, ya que el follaje reduce el impacto de las gotas de lluvia, disminuye la fricción del escurrimiento superficial y con ello su poder erosivo, evitando la pérdida de millones de toneladas de suelo productivo, principalmente de terrenos agrícolas de ladera.

\section{Literatura citada}

Alcalá-de-Jesús, M., Oropeza-Mota, .J.L., y Tovar-Salinas, J.L. 1998. Producción de sedimentos en diferentes usos del suelo, en la cuenca del Río los Sauces, Michoacán. Agrociencia, 32: 225-231.

Belmonte S., F., A. Romero D. y F. López B.

1998. Efectos sobre la cubierta vegetal, la escorrentía y la erosión del suelo, de la alternancia cultivo-abandono en parcelas experimentales. Investigaciones geográficas, 22 : 95-107

Betancourt Y., P., J. L. Oropeza M., B. Figueroa S., V. Ordaz C., C. Ortiz S. y A. Hernández G.

2000. Pérdidas de suelo y potencial hidrológico en parcelas con coberturas vegetativas de especies forrajeras. Terra, 18 : 263-275.

Blackburn, W.H., Pierson, F.B., Hanson, C.L., Thurow, T.L., \& Hanson, A.L.

1992. The Spatial and Temporal Influence of Vegetation on Surface Soil Factors in Semiarid Rangelands. Transactions of the ASAE, 35: 479-486.

Carroll, C., Merton, L. \& P. Burger.

2000. Impact of Vegetative and Slope on Runoff, Erosion, and Water Quality for Field Plots on a Range of Soil and Spoil Materials on Central Queensland Coal Mines. Australian Journal of Soil Research, 38: 313-327.

Carroll, C., M. Halpin, P. Burger, K. Bell, M. M. Sallaway and D. F. Yule.

1997. The Effect of Crop Type, Crop Rotation, and Tillage Practice on Runoff and Soil Loss on a Vertisol in Central Queensland. Australian Journal of Soil Research, 35: 925-939.

Eastman, J.R.

2003. IDRISI: Guide to GIS and Image Processing. Version 14.0 (Kilimanjaro). Clark University.

Espinosa, R.M., Andrade, LE., Rivera, OP., Romero D.A.

2011. Degradación de suelos por actividades antrópicas en el norte de Tamaulipas, México. Papeles de Geografía, 53-54: 77-88.
Faminow, M.D.

1998. Cattle, Deforestation and Development in the Amazon: An Economic, Agronomic and Environmental Perspective. FAO.

CAB International Wallingtord. U.K. 253 p.

2000. Manual de prácticas integradas de manejo y conservación de suelos. Boletín de tierras y aguas de la FAO. Roma. Italia. $220 \mathrm{p}$.

Flores, L. H. E., Mora, O.C., Ruiz, C.J.A., Chávez, D.A.A.

2013. Efecto de la cobertura de suelo de tres cultivos sobre la erosión hídrica. Revista Chapingo serie zonas áridas (RCHSZA). XII (1): 19-25.

Francisco-Nicolás, N., Turrent-Fernández, A, Oropeza-Mota, J.L., Martínez-Menes, M.R., Cortés-Flores, J.I.

2006. Pérdida de suelo y relación erosión-productividad en cuatro sistemas de manejo del suelo. Terra Latinoamericana, 24 (2): 253-260.

Gutierrez, J. \& Hernandez, I. I.

1996. Runoff and Interrill Erosion as Effected by Grass Cover in a Semi-arid Rangeland of Northern Mexico. Journal of Arid Environments, 34: 287-295.

Lal, R.

2000. Integrated Watershed Management in the Global Ecosystem. Edited by Rattan Lal. Soil and Water Conservation Society. New York: CRC Press. 395 p.

Loch, R. J.

2000. Effects of Vegetation Cover on Runoff and Erosion under Simulated Rain and Overland Flow on a Rehabilitated Site on the Meandu Mine, Tarong, Queensland. Australian Journal of Soil Research, 38: 299-312.

Osuna, C. E. S., y Esquivel V. F.

1996. Cobertura vegetal y erosión del suelo en Aguascalientes. Agricultura Técnica en México, 22: 127-144.

Quinton, J. N., Edwards, G. M. and Morgan, R. P. C. 1997. The Influence of Vegetation Species and Plant Properties on Runoff and Soil Erosion: Results from a Rainfall Simulation Study in South East Spain. Soil Use and Management, 13: 143-148. 
Ramírez C., M. E. y J. L. Oropeza M.

2001. Eficiencia de dos prácticas productivo conservacionistas para controlar erosión de laderas en el trópico. Agrociencia, 35: 489-495.

Ríos, B. J. D. y Martínez, M. M. R.

1990. Efecto de la cobertura vegetal en el proceso erosivo. Agrociencia, 1: 61-73.

Rivera-Ruíz, P., Oropeza-Mota, J.L., Martínez-Menes, M.R., Mejía-Sáenz, E., Tapia-Vargas, L.M., Ventura-Ramos, E. Jr. 2012. El proceso lluvia-escurrimiento-erosión en laderas y microcuencas instrumentadas. Tecnología y Ciencias del Agua, III (4): 151-166.

Santacruz, De L.G.

2011. Estimación de la erosión hídrica y su relación con el uso del suelo en la cuenca del río Cahoacán, Chiapas, México. Aqua-LAC, 3 (1): 45 - 54
Serna, P.A. y Echavarría, C. F. G.

2002. Caracterización hidrológica de un agostadero comunal excluido al pastoreo en Zacatecas, México. I. Pérdidas de suelo. Técnica Pecuaria México, 40: 37-53.

Solano, T. J. A., Martínez, M. M., Oropeza, M. J. L. y Anaya, G. M. 1990. Efecto de la relación precipitación-escorrentía en el proceso erosivo en diferentes usos de suelo en la cuenca del Río Texcoco. Agrociencia, 1: 25-44.

Wilcox, B. P., \& Wood, M. K.

1989. Factors influencing interrill erosion from semiarid slopes in New Mexico. Journal of Range Management, 42: $66-70$ 